\title{
Guest editorial: special issue on spatial computing in emergency management
}

\author{
Hui Zhang ${ }^{1} \cdot$ Yan Huang ${ }^{2} \cdot$ Jean-Claude Thill ${ }^{3}$ • \\ Danhuai Guo ${ }^{4}$ Yi Liu ${ }^{5}$
}

Published online: 6 April 2018

(C) Springer Science+Business Media, LLC, part of Springer Nature 2018

This special issue was conceived by the guest editors with the goal of providing a synthesis of the state of the art of research involving spatial computing in emergency management. Emergency management (EM) has for the past two decades been at the cutting edge of developments in geographic information science and geospatial technologies for several core reasons. First, EM has an obvious spatial dimension because emergencies affecting people and organizations, individually and collectively, occur at locations scattered across space and risk is spatially distributed, while assets mobilized for mitigation, preparedness, response, and recovery are at discrete locations. Second, EM is multidimensional, involving the natural realm (esp. water and atmospheric conditions), human and social realities (including behaviors and emotions), and infrastructures. Also, compounding the complexity of spatial computing is that

Jean-Claude Thill

Jean-Claude.Thill@uncc.edu

Hui Zhang

zhhui@mail.tsinghua.edu.cn

Yan Huang

huangyan@unt.edu

Danhuai Guo

guodanhuai@cnic.cn

Yi Liu

liuyi@tsinghua.edu.cn

1 Tsinghua University, Beijing, China

2 University of North Texas, Denton, TX, USA

3 University of North Carolina at Charlotte, Charlotte, NC, USA

4 Computer Network Information Center, Chinese Academy of Sciences, Beijing, China

5 Institute of Public Safety Research, Tsinghua University, Beijing, China 
EM involves space-time relationships spanning broad sections of society's socio-physical systems. Because EM situations are hazard-driven, uncertain, and extreme, spatial computing must be resilient, quasi-realtime, massive, and capable of integrating structured as well as unstructured data. EM can be seen as the epitome of wicked problems, a class of problems that are recognized to defy commonplace solution strategies .

The selection of 8 papers published in this special issue is broadly representative of the most recent developments in spatial computing in situations of emergency management. Following an open call for papers disseminated on various platforms, 12 papers were submitted for possible publication. All the submissions were handled through the customary peer-review process involving a number of internationally renowned scholars.

The first paper entitled 'The National Geographic Characteristics of Online Public Opinion Propagation in China Based on WeChat Network' written by Ai et al. analyzes a large dataset obtained from the most popular social media application in China and constructs a spatial interaction network to uncover the geographic interaction characteristics of online public opinion propagation.

In 'Efficient Task Assignment in Spatial Crowdsourcing with Worker and Task Privacy Protection', Liu et al. identify mutual privacy protection requirements in the course of spatial crowdsourcing and present a privacy-preserving task assignment protocol for protecting the privacy for both workers and task requesters while maintaining the functionality of SC systems.

The paper entitled 'Spatio-temporal Prediction of Crop Disease Severity for Agricultural Emergency Management Based on Recurrent Neural Networks' written by Xu et al. proposes a novel crop disease prediction framework based on ensemble learning techniques and spatio-temporal recurrent neural networks for agricultural emergency management.

The article by Guo et al. on 'OSCAR: A Framework to Integrate Spatial Computing Ability and Data Aggregation for Emergency Management of Public Health' proposes a comprehensive framework for spatial analysis on public health issues. The developed framework provides a data resource aggregation platform to help find newly occurred cases of diseases from different regions and deploys a series of computation images in a cloud-computing environment to shorten the computation time of the algorithms.

Another paper, 'A Spatio-temporal Scenario Model for Emergency Decision', written by Liu et al. develops a general scenario model for scenario-based emergency decision-making. The developed model proposes a structural and quantitative representation for disaster status, and employs a qualitative spatial representation model named grid-based hierarchical qualitative spatial representation and calculation model to show the spatial relation of scenarios.

Cui et al. study emergency traffic evacuation in their paper on 'Multi-Vehicles Dynamic Navigating Method for Large-Scale Event Crowd Evacuations' by introducing the state of each road link in a routable network. The model is established on the basis of the theory of spatial diversity and the theory of minimum cost maximum flow. An empirical use case is also presented.

In the article 'Close Range Photogrammetry with Tablet Technology in Post-earthquake Scenario: Sant'Agostino Church in Amatrice', Dabove et al. deal with the use of tablet devices for rapid close range photogrammetry in post-earthquake scenario, following the close range photogrammetry approach, and apply the technique to the case study of the Sant'Agostino Church in Amatrice, Italy.

Finally, in 'Efficient CPS Model Based Online Opinion Governance Modeling and Evaluation for Emergency Accidents' Deng et al. propose a new online opinion governance system 
based on a cyber-physics-society (CPS) Model that is constructed on a cell phone application for data collection including geospatial information and online opinion.

This special issue was preceded by an international workshop on Emergency Management using GIS, held in San Francisco, CA in October 2016 in conjunction with the annual ACM SIGSPATIAL conference. Contributions included in this special issue span a range of cuttingedge topics involving spatial computing in the context of emergency management. These contributions incorporate various information technologies and call upon a number of systems designs and algorithmic structures that will appeal to scholars in geospatial sciences as well as information technology practitioners involved in the emergency management sphere. 